\title{
Cancer vaccine strategies get bigger and better
}

\begin{abstract}
Many anti-tumor vaccines have failed in recent clinical trials because they included the wrong patients and the wrong immune adjuvants - and little was learned because the anti-tumor immune response was not carefully monitored. A new tumor vaccine for lymphoma sets the stage for how future trials should be carried out (pages 1171-1177).
\end{abstract}

$\mathrm{T}$ HE DISCOVERY OF distinct antigenic features of neoplastic cells has suggested that it may be possible to create antitumor vaccines ${ }^{1}$. Vaccine strategies have used tumor-derived cells ${ }^{2}$, or cellular material $^{3}$, containing cryptic or unidentified antigens to induce specific immune recognition of these tumor-associated antigens (TAAs). Alternatively, several recently discovered tumor-associated antigens have been tested directly as immunogens in vaccine formulations ${ }^{4,5}$. To improve antigen presentation, vaccine approaches have also included adjuvant agents to potentiate immune recognition of the TAA. These adjuvants have evolved to improve immune activation, and are the result of many years of research directed towards understanding of the mechanisms of anti-tumor immunity ${ }^{6}$. Bendandi et al., on page 1171 of this issue ${ }^{7}$, report a TAA vaccination protocol that, with granuloyte-monocyte colony-stimuting factor (GMCSF) as an immune adjuvant, achieves an effective antitumor response.

A variety of anti-tumor vaccine clinical trials have been recently undertaken. In spite of the large number of these trials, and the plethora of distinct approaches investigated, there has been little evidence of clinical efficacy, ${ }^{3,4}$ Furthermore, precise correlates of clinical effects and immunological responses have been lacking. These limitations reflect several shortcomings in the design of the studies. Studies have often used cohorts of patients with advanced stage disease, for which a full response is unlikely for various biological reasons. Moreover, many of the adjuvants tested lacked the potential to effectively overcome intrinsic immunosuppressive activity of the tumor. Finally, precise assays to monitor clinical response and, most importantly, immune responses have been lacking. Thus, despite a large body of work in this area, many tumor vaccine protocols tested to date have not

\section{By Tanja D. De GruijL ${ }^{1} \&$ DAVID T. CURIEL ${ }^{2}$}

proven effective or yielded large amounts of useful information.

The study by Bendandi et al. makes a large advance over previous tumor vaccine trials. Idiotypic determinants of the Ig synthesized by a clonal B-cell cancer, such as follicular lymphoma (FL) are unique, and are thus useful as TAAs (refs.

cell priming. The vaccine was administered in conjunction with the molecular adjuvant GM-CSF, which is very effective in induction of immune effector cells $s^{6,8}$. The protocol also included highly sensitive assays to carefully monitor detectable translocations in the blood and quantify the tumor-specific T-cell response, the hallmark of true anti-tumor immunization.

This study included 20 FL patients, eleven of which possessed the chromosomal translocations characteristic of FL. After six months recovery from chemotherapy, patients were vaccinated with four monthly injections of their own lymphoma Ig idiotypic determinant, along with recombinant GM-CSF. After vaccination, patients were monitored for chromosome translocation-bearing cells by PCR. Anti-tumor immunity was determined by assays to measure cytolysis and cytokine release in response to autologous tumor cells. Of the eleven patients evaluated, eight cleared the residual chromosomal translocation-bearing cells in response to vaccination. Nineteen of the twenty patients tested showed specific $\mathrm{CD}^{+}$and $\mathrm{CD} 4^{+} \mathrm{T}$-cell reactivity to the tumor. Evidence of antitumor immunity correlated with clinical evidence of a reduction of tumor burden.

This trial, along with other

$4,8,9)$. The vaccine strategy of Bendandi $e t$ al. included a new group of follicular lymphoma (FL) patients that were in complete remission after chemotherapy. Most FL patients in remission still have chromosomal translocation-bearing tumor cells, and are therefore at risk for relapse. The authors created a vaccine that combined an FL-associated idiotype IgG with keyhole limpet hemocyanin (KLH) (Fig. $1)$. The inclusion of KLH provided additional epitopes for T-helper-cell recognition, allowing for optimal CTL activation, and also provided a positive control antigen, allowing the authors to monitor T- recent trials, has benefited from the advances made in our understanding of anti-tumor immunity. Most new immunotherapeutic approaches aim to promote the activation of $\mathrm{CD}^{+}$cytotoxic $\mathrm{T}$ lymphocytes (CTLs), which have been recognized as the main effector cells in a multitude of murine tumor rejection models. It is also known that tumors use a variety of mechanisms to induce host tolerance and prevent TAA-specific CTL activation $^{6}$. Dendritic cells (DCs), which express high levels of MHC molecules, co-stimulatory molecules, and T-cell-activating cytokines, may be useful in over- 
coming these obstacles and activating TAA-specific CTL responses (Fig. 1). Many new vaccines, therefore, also include autologous DCs that have been loaded with TAA (refs. 3,4,6) in vitro. This study instead uses the DC stimulant GMCSF.

GM-CSF functions to promote TAA presentation by antigen presenting cells (APCs) and CTL activation by recruiting peripheral DC precursors and inducing DC maturation (Fig. 1) (ref. 6). Recent studies have also confirmed the role of CD40-mediated activation of DCs in activation of the TAA-specific CTL response $^{10}$. Indeed, CTL-dependent rejection of B-lymphomas in mice was recently reported after treatment with an agonistic antibody against CD40 (ref. 11). Thus, GM-CSF and CD40 ligand may prove to be a winning adjuvant combination in future lymphoma, as well as other tumor vaccines.

The vaccine strategy reported by Bendandi et al. does not represent a particularly new approach, but its validation of therapeutic benefit, well-planned study design, and careful execution allow this study to stand as a hallmark in the field of cancer vaccine development. Consideration of the patients' disease stage cannot be ignored in determining the potential of a vaccine to achieve a beneficial therapeutic outcome. These findings suggest that in cancer therapy, early intervention with vaccine-based approaches is warranted. Furthermore, studies including specific TAAs as vaccines should carefully monitor post-vaccination immune responses. Future studies aimed at identification of TAAs for other tumor types, and additional carefully- monitored tumor vaccine trials, should lead to the development of new, more effective vaccine strategies.

1. Rosenberg, S.A. A new era for cancer immunotherapy based on the genes that encode cancer antigens. Immunity 10, 281-287 (1999).

2. Vermorken, J.B. et al. Active specific immunotherapy for stage II and stage III human colon cancer: a randomized trial. Lancet 353, 345-350 (1999).

3. Nestle, F.O. et al. Vaccination of melanoma patients with peptide- or tumor lysate-pulsed dendritic cells. Nature Med. 2, 52-58 (1998).

4. Hsu, F.J. et al. Vaccination of patients with B-cell lymphoma using autologous antigen-pulsed dendritic cells. Nature Med. 2, 52-58 (1996).

5. Rosenberg, S.A. et al. Immunologic and therapeutic evaluation of a synthetic peptide vaccine for the treatment of patients with metastatic melanoma. Nature Med. 4, 321-327 (1998).

6. Pardoll, D.M. Cancer vaccines. Nature Med. (suppl.) 4, 525-531 (1998)

7. Bendandi, et al. Molecular complete remissions induced by patient-specific vaccination plus GMCSF against lymphoma. Nature Med. 5, 1171-1177 (1999).

8. Levitsky, H.I. et.al. Immunization with granulocyte-macrophage colony stimulating factor-transduced, but not B7.1-transduced, lymphoma cells primes idiotype-specific T cells and generates potent systemic antitumor immunity. J. Immunol. 156, 3858-3865 (1996)

9. Nelson, E.l. et al. Tumor-specific, cytotoxic T-lymphocyte response after idiotype vaccination for Bcell, non-Hodgkin's lymphoma. Blood 88, 580-589 (1996)

10. Mackey, M.F. et al. Dendritic cells require maturation via CD40 to generate protective antitumor immunity. J. Immunol. 161, 2094-2098 (1998).

11. French, R.R. et al. CD40 antibody evokes a cytotoxic $T$-cell response that eradicates lymphoma and bypasses T-cell help. Nature Med. 5, 548-553 (1999).

${ }^{1}$ Department of Pathology and Medical Oncology University Hospital Vrije Universiteit

1007 MB Amsterdam, The Netherlands

${ }^{2}$ Gene Therapy Center

The University of Alabama at Birmingham,

$18246^{\text {th }}$ Avenue South

Birmingham, Alabama 35294-8627, USA

E-mail:david.curiel@ccc.uab.edu

\title{
Unraveling hepatitis B virus infection of mice and men (and woodchucks and ducks)
}

\author{
The development of liver diseases in patients suffering from chronic hepatitis B infection is determined by the \\ nature of the host immune response. Several factors have made this immune response difficult to study, \\ but recently developed animal models will help clear the way.
}

NFECTION OF ADULT humans with the hepatitis B virus (HBV) characteristically results in self-limited disease and in clinical recovery. Persistent or chronic infection is an outcome that usually accounts for less than $5 \%$ of adult HBV infections. Children often develop persistent infection, which later in life causes chronic hepatitis, cirrhosis of the liver, and, in some, primary hepatocellular carcinoma (HCC). Although safe and effective HBV vaccines are now available, over 300 million people worldwide are chronically infected with $\mathrm{HBV}$, and these individuals have a high relative risk of developing life-threatening liver disease.

Substantial evidence now suggests that differences in outcome of HBV infection are determined by variations in the immune response. Cell-mediated immunity involving T-helper (Th) cells and cytotoxic $\mathrm{T}$ lymphocytes (CTL) determines whether HBV infection is followed by recovery or viral persistence (Fig. 1). During acute self-limited virus infection, patients

\section{STEPHAN MENNE \& Bud C. TENNANT}

show vigorous, polyclonal Th and CTL responses against epitopes of viral capsid and envelope proteins ${ }^{1}$, leading to the clearance of viral DNA and envelope proteins from the serum. Patients who develop chronic HBV infection have been reported to have less-vigorous Th responses, characterized by low frequency of HBV-specific Th cells in the peripheral blood and the liver, and weak or undetectable virus-specific CTL responses ${ }^{1}$. A weak CTL response is also believed to cause the hepatic injury that occurs in chronically infected patients.

The immunopathogenesis of HBV infection has been difficult to investigate. HBV has a narrow host range limited to endangered animal species. Tissue culture systems used to study most other viral pathogens are not yet available for HBV.
The original studies of HBV immunopathogenesis were limited to infected patients at varying stages of disease following accidental exposure, and to experimentally infected chimpanzees ${ }^{1,2}$.

In recent years, new animal models of HBV infection have contributed substantially to our understanding of this virus ${ }^{3}$. The woodchuck (Marmota monax) and the Pekin duck (Anus domesticus) both have naturally occurring infections with viruses that belong to the family Hepadnaviridae, of which HBV is the prototype virus. Woodchucks with naturally or experimentally acquired, persistent woodchuck hepatitis virus infection develop chronic hepatitis and HCC similar to those associated with HBV infection. Duck hepatitis B virus, is not typically associated with liver disease. Although both the duck and the woodchuck have been valuable in molecular studies of viral replication and have been useful in the preclinical evaluation of prospective antiviral 